\title{
CONSOMMATION ALIMENTAIRE DE PONDEUSES Ce ET ce
}

\author{
P. MÉRAT \\ avec la collaboration technique de $A$. Bordis \\ Station centrale de Génétique animale \\ Centre national de Recherches zootechniques, 78 - Jouy-en-Josas \\ Institut national de la Recherche agronomique
}

Dans une expérimentation sur 53 pondeuses d'un an en cages individuelles (PROD'homme et MÉrat, I965), les poules blanches cc avaient eu une consommation alimentaire "résiduelle " (corrigée pour le poids corporel, sa variation et le poids d'œufs pondus) inférieure de 4 p. roo à celle de leurs sœurs colorées Cc. Des différences hautement significatives existaient aussi entre familles pour ce cuitère.

En refaisant un essai similaire, nous avons obtenu des résultats du même ordre, que nous allons décrire brièvement.

De mai à juillet I 967 ( 3 périodes de 28 jours), 62 pondeuses âgées de I I mois, appartenant à 6 familles de sœutrs de notre troupeau, ont été mises en cages individuelles. Dans chaque famille, le même nombre de poules $\mathrm{Cc}$ et cc était gardé.

Tous les 28 jours, les bêtes étaient pesées, ainsi que leurs œufs et l'aliment ingéré, une correction individuelle était faite pour le gaspillage $\left(^{*}\right)$. L'aliment était donné sous forme de farine. Il correspondait à la formule normale "reproducteurs " de notre élevage, à $\mathrm{i} 6 \mathrm{p}$. Ioo de protéines.

\section{Mesures "brutes"}

Les valeurs moyennes sur l'ensemble des trois périodes sont données, pour chaque facteur, dans le tableau I. Elles portent sur 48 poules dont toutes les performances étaient enregistrées dans les trois périodes.

Une analyse de variance sur ces mesures indique un effet du génotype au locus C significatif au seuil 5 p. Ioo pour le poids d'œufs pondus et la consommation d'aliment, mais non pour le poids moyen et le gain de poids (1). Quant aux différences entre familles, elles n'atteignent le seuil $5 \mathrm{p}$. Ioo de signification que pour le gain de poids.

(*) Ce dernier présentait aussi des différences faniliales significatives au seuil I p. 100.

(1) Toutefois, comme en 1965 , le gain de poids est inférieur pour les poules cc. Il serait souhaitable de voir si, dans ces conditions, la persistance de la ponte sur une longue durée est aussi bonne pour ces dernières que pour leurs sœurs colorées. 
TABLEAU I

Moyenne des performances "brutes" pir génotype

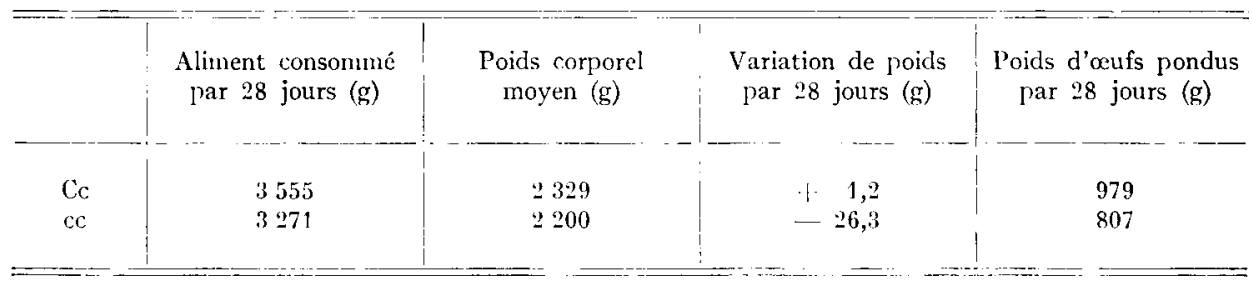

2. Mesures "corrigées"

D'après la méthode de BYERLY (I94I), nous avons établi les équations de régression multiple permettant d'estimer la consommation théorique d'une poule sur une durée de 28 jours en fonction de son poids, de sa variation de poids et du poids d'œufs pondus (ProD'homme, I965). Ces équations sont les suivantes, pour les génotypes $\mathrm{Cc}$ et $\mathrm{cc}$ respectivement (avec $\mathrm{A}=$ quantité d'aliment consommée, $\mathrm{P}=$ poids corporel, $\mathrm{DP}=$ variation de poids, $\mathrm{O}=$ poids d'œufs) :

$$
\begin{aligned}
& \mathrm{A}=55,6 \mathrm{P}^{0^{5}}+\mathrm{I}, 97 \mathrm{DP}+0,85 \mathrm{O} \\
& \mathrm{A}=60,9 \mathrm{P}^{0^{5}}+\mathrm{I}, 28 \mathrm{DP}+0,66 \mathrm{O}
\end{aligned}
$$

Les différences entre les coefficients ne sont pas significatives. Cependant, on peut noter qu'elles vont dans le même sens qu'en I965: le coefficient de DP et celui de $\mathrm{O}$ sont un peu inférieurs pour les poules cc.

A partir d'une équation de régression commune, nous avons calculé pour chaque poule la différence entre sa consommation réelle et sa consommation théorique exprimée sn écart à la moyenne générale. La moyenne de ces différences est $+97,3$ pour les animaux $C c$ et $-29,9$ pour les cc. Comme le montre le tableau 2, la différence entre génotypes s'approche du niveau $5 \mathrm{p}$. Ioo de signification. Les écarts entre familles ne sont pas significatifs dans notre échantillon, non plus que l'interaction génotype $\times$ famille.

\section{TABLEAU 2}

Analyse de variance pour la consommation "corrigée "

\begin{tabular}{|c|c|c|c|c|}
\hline $\begin{array}{c}\text { Source } \\
\text { de variation }\end{array}$ & $\begin{array}{c}\text { Degrés } \\
\text { de liberté }\end{array}$ & Variance & $\mathrm{F}$ & Signification \\
\hline Génotypes & 1 & $1 / 4489$ & 2,81 & $\mathrm{P}<0,10$ \\
\hline Familles . & 5 & 75141 & 1,47 & N.S. \\
\hline Interaction ... & 5 & $7355^{\prime} \mathbf{x}$ & $1,1 / 4$ & N.S. \\
\hline Résiduelle .... & 50 & 50986 & - & - \\
\hline
\end{tabular}

(Différence entre consommation observée et théorique, en grammes, sur 28 jours)

Comme dans notre première expérience (ProD'homme et Mŕrat, I965), les poules $\mathrm{Cc}$, à en juger par leur consommation " corrigée ", sont moins bonnes utilisatrices que leurs sœurs $c c$, à production, gain de poids et poids égaux. La différence 
(significative dans la première expérience et sur l'ensemble des données) représente ici 3 p. Ioo environ de la consommation totale, contre $4 \mathrm{p}$. Ioo en I 665 . Nos premières observations paraissent donc bien confirmées.

Reçu pour publication en mai 1968.

\section{SUMMARY}

FOOI CONSUMPTION OF (Cc) AND $(c c$ ) LAYING IIENS

62 one year-old laying hens, coloured $(\mathrm{C} c)$ or " recessive white " $(c c)$, equally distributed in 6 fullsister families, were tested in cages for a 3 -month period.

For each hen, the deviation of the observed food consumption for a 28 -day period to the multiple regression of food consumption on weight, weight gain or loss, and total weight of eggs laid, (according to BYERLY's method) was derived. For white $(c c)$ hens, this deviation was lower than for coloured ones, the difference in " corrected " consumption being about 3 per cent. This is in agreement with a previous result.

\section{RÉFÉRENCES BIBLIOGRAPHIQUES}

Byerty T. C., I94I. Feeds and other costs of producing market eggs. Univ. Maryland Agric. Exp. Stat. Bulletin $n^{\circ} \mathrm{A}$ I.

Prod'homise J., 1965. Calcul d'une équation penmettant de prévoir la consommation alimentaire de pondeuses en fonction de leurs performances. Ann. Zootech., 14, 335-339.

Prod'iomme J., Mérat P., 1965. Consommation alinentaire chez des pondeuses de génotypes Cc et cc issues de plusieurs familles. Ann. Zootech., 14, $34 \mathrm{I}-35^{\circ}$. 